Jeanne-Marie Boivin, Naissance de la fable en français. L'Isopet de Lyon" et l'"Isopet I-Avionnet"

\title{
Paola Cifarelli
}

\section{(2) OpenEdition}

1 Journals

\section{Édition électronique}

URL : http://journals.openedition.org/studifrancesi/9426

DOI : 10.4000/studifrancesi.9426

ISSN : 2421-5856

Éditeur

Rosenberg \& Sellier

\section{Édition imprimée}

Date de publication : 1 décembre 2007

Pagination : 626-627

ISSN : 0039-2944

\section{Référence électronique}

Paola Cifarelli, "Jeanne-Marie Boivin, Naissance de la fable en français. L"'Isopet de Lyon" et I" Isopet I-

Avionnet" », Studi Francesi [En ligne], 153 (LI | III) | 2007, mis en ligne le 30 novembre 2015, consulté le 12 janvier 2021. URL : http://journals.openedition.org/studifrancesi/9426 ; DOI : https://doi.org/

10.4000/studifrancesi.9426

Ce document a été généré automatiquement le 12 janvier 2021.

\section{(c)}

Studi Francesi è distribuita con Licenza Creative Commons Attribuzione - Non commerciale - Non opere derivate 4.0 Internazionale. 


\title{
Jeanne-Marie Boivin, Naissance de la fable en français. L" Isopet de Lyon" et l'"Isopet I-Avionnet"
}

\author{
Paola Cifarelli
}

\section{RÉFÉRENCE}

JEANNE-MARIE BOIVIN, Naissance de la fable en français. L"Isopet de Lyon" et l'“Isopet I-

Avionnet”, Paris, Champion, 2007 («Essais sur le Moyen Âge» 33), pp. 499.

1 Ce beau volume constitue une contribution d'un grand intérêt, non seulement pour les spécialistes de la tradition ésopique, mais pour les études médiévales dans leur ensemble. En effet, si les textes des Isopets sont disponibles depuis longtemps en édition moderne grâce aux travaux de Julia Bastin et de Pierre Ruelle, la critique a négligé le genre de la fable en ancien français, qui jusqu'ici n'avait pas fait l'objet d'une étude systématique.

2 Dans ce travail, l'A. présente les premiers fruits de ses recherches, portant sur les deux collections qui furent traduites à partir de l'Anonymus Neveleti; ils seront complétés par un deuxième tome, à paraître prochainement. Ici, deux grands noyaux thématiques sont pris en considération successivement: dans la première partie («Gestations: les fables latines»), les différentes traditions latines qui sont à la base des recueils français sont l'objet d'une fine analyse critique et stylistique, prémisse indispensable pour toute étude concernant un genre qui se veut fortement ancré à la tradition; quant à la deuxième partie («Naissance: les Isopets»), elle a pour objet les deux collections françaises, envisagées selon la perspective des sources, des auteurs et du public, ainsi que de la poétique qui les sous-tend.

3 Les quatre chapitres de la première partie sont consacrés chacun aux différentes collections qui sont à la base du corpus auquel puisent les translateurs médiévaux. Après une première enquête sur la fable-épigramme de la collection phédrienne et sur 
la fable élégiaque telle qu'elle a été conçue par Avianus avec un talent parfois comparable à celui de La Fontaine, J.M.B. examine les répertoires de motifs ésopiques contenus dans les différents Romulus en prose, dont on sait l'importance au Moyen Âge même en dehors du genre de la fable proprement dit. Enfin, le quatrième chapitre porte sur l'Anonymus Neveleti, collection qui est à la base des deux Isopets analysés dans la seconde partie. La méthode suivie par l'A. dans cette section lui permet d'examiner sous plusieurs points de vue la tradition de ce recueil latin à succès, dont on conserve quelque deux cents manuscrits et une trentaine d'incunables; après avoir abordé les problèmes épineux liés à l'identité du compilateur et aux circonstances de composition, l'attention se fixe sur les caractéristiques stylistiques du recueil, véritable «exercice de style» (p. 162) dont la virtuosité technique et verbale trahit son utilisation dans le cursus de rhétorique.

La deuxième partie s'ouvre sur une réflexion à propos des titres des recueils en français et sur l'utilisation du diminutif; la question qui sous-tend cette analyse est plus vaste, et concerne le milieu dans lequel les textes en langue vernaculaire se diffusèrent. L'analyse de J.M.B., portant également sur la matérialité des codex, l'analyse des prologues, les recherches autour des dédicataires et propriétaires des fabliers, montre que si certains indices pourraient faire pencher pour une utilisation scolaire, en réalité il faut plutôt penser à une diffusion dans le milieu curial, et tout spécialement parmi le public féminin. Dans ce contexte, la présence du texte latin à coté du français dans certains manuscrits est très opportunément considérée comme une «valeur ajoutée» destinée à augmenter le prestige d'un genre considéré mineur.

5 L'étude ponctuelle des textes et des codex amène l'A. à formuler d'importantes observations sur plusieurs points. Nous nous bornerons à citer ici l'étude des différences qui séparent les deux rédactions de l'Isopet-I, que le lecteur de l'édition critique avait beaucoup de peine à entrevoir, ainsi que les recherches sur les auteurs des recueils de fables; à ce propos, si l'examen des textes est destiné à laisser dans l'ombre l'identité des fabulistes, l'attention pour des données parallèles et le flair du chercheur raffiné qu'est J.M.B. permettent à celle-ci de parvenir à l'identification du remanieur de la deuxième version de l'Isopet-I (le fruit de ces recherches avait été anticipé dans la «Romania» CXXIII, 2005, pp. 459-85, cfr. le fasc. 147 de ces «Studi»).

Par-delà la fine analyse consacrée aux spécificités de chacun des deux recueils français qui font l'objet de cette étude, et rendant compte des critères de sélection des motifs, des qualités propres au style de chaque translateur-adaptateur ainsi que de la physionomie des traductions, les différents chapitres de la seconde partie permettent de dégager des principes généraux valables pour tout recueil de fables en français: à savoir, que les fabulistes opèrent toujours, plus ou moins consciemment, des contaminations avec des versions parallèles d'un même motif et, élément non moins important, que chaque fablier est le produit du milieu d'où il sort: la culture dont il est porteur influence donc fortement le lexique, le style, les idées véhiculées par chaque texte.

7 Dans le chapitre final, l'A. aborde des questions liées à la 'forme' des fables, dans ses deux parties (récit et morale); prélude au deuxième volume en préparation, ces pages mettent en lumière les rapports qui lient ces deux éléments de l'apologue dans les fabliers français.

8 Ce travail si riche et dense, qui permet de jeter enfin la lumière sur les deux textes médiévaux connus par La Fontaine, n'est pas moins utile dans ses sections 
complémentaires. Celles-ci comprennent des tables des concordances des fables dans les différentes versions, les statistiques sur la longueur des apologues ainsi que celles à propos des différentes typologies de conclusion moralisante; elles fournissent un matériau complémentaire que tout chercheur travaillant sur la fable pourra utiliser avec profit. Il en va de même pour la section bibliographique, qui constitue non seulement le gage de la solidité de cette étude, mais aussi un instrument incontournable pour tous ceux qui voudront se pencher sur ce genre dans le futur.

9 On ne peut donc que féliciter l'A. de ce volume, qui représentera assurément un point de repère critique indispensable. 